Friedrich Buchmayr

\title{
Einblicke in klösterliche Archive und Bibliotheken am Beispiel von St. Florian
}

In Österreich gibt es derzeit 190 Ordenshäuser mit rund 5.800 Ordensleuten (vgl. www.ordensgemeinschaft.at). Viele Stifte und Klöster blicken auf eine jahrhundertelange Geschichte zurück und beherbergen reichhaltige Bibliotheken und Archive, die für die österreichische Kulturgeschichte von großer Bedeutung sind. Es ist nicht möglich, in der gebotenen Kürze auch nur einen Überblick über diese höchst vielfältigen Sammlungen zu geben. Deshalb wird hier der Weg gewählt, exemplarisch ein einzelnes Stift, nämlich das Augustiner-Chorherrenstift St. Florian, in den Mittelpunkt zu stellen und Einblick in die Stiftsbibliothek (Abb. 1) und das Stiftsarchiv dieses Hauses zu geben.

\section{Kurzgeschichte des Stiftes St. Florian}

Das Stift St. Florian kennt keinen Gründer, dürfte aber schon Ende des 8. Jahrhunderts existiert haben. Die Ordensgeistlichen orientierten sich vermutlich an der Aachener Regel (816). Um 1071 erfolgte die Neubesiedelung des Klosters durch Augustiner-Chorherren, die bis zum heutigen Tag das Stift und die dazugehörigen 33 Stiftspfarren betreuen (vgl. Rehberger 2009, 6-8; Reisinger und Brandes 1997, 337-384).

Auf die kunst- und architekturhistorische Bedeutung des Stiftes St. Florian kann hier nur kurz verwiesen werden (vgl. Holzinger und Buchmayr 2009). Im Mittelalter wirkte hier eine Schreib- und Malerschule, die führend und stilbildend im Donauraum war. Es entstanden Meisterwerke wie die Riesenbibel von St. Florian (um 1140; vgl. Buchmayr et al. 2008), eine frühe und qualitätsvolle Biblia pauperum (um 1310) und eine Reihe prächtig illuminierter hochgotischer Handschriften für den liturgischen Gebrauch (vgl. Holter 1988; Schmidt 1962).

Das ab 1686 umgestaltete und erweiterte Klostergebäude mit Stiftskirche, Marmorsaal und Kaiserzimmern zählt zu den eindrucksvollsten Barockanlagen Österreichs (vgl. hierzu Korth 1975, 2009a und 2009b). Die große Orgel der Stiftsbasilika erlangte durch den berühmtesten Stiftsorganisten, den Komponisten Anton Bruckner, internationale Berühmtheit (vgl. Rehberger et al. 1998). 


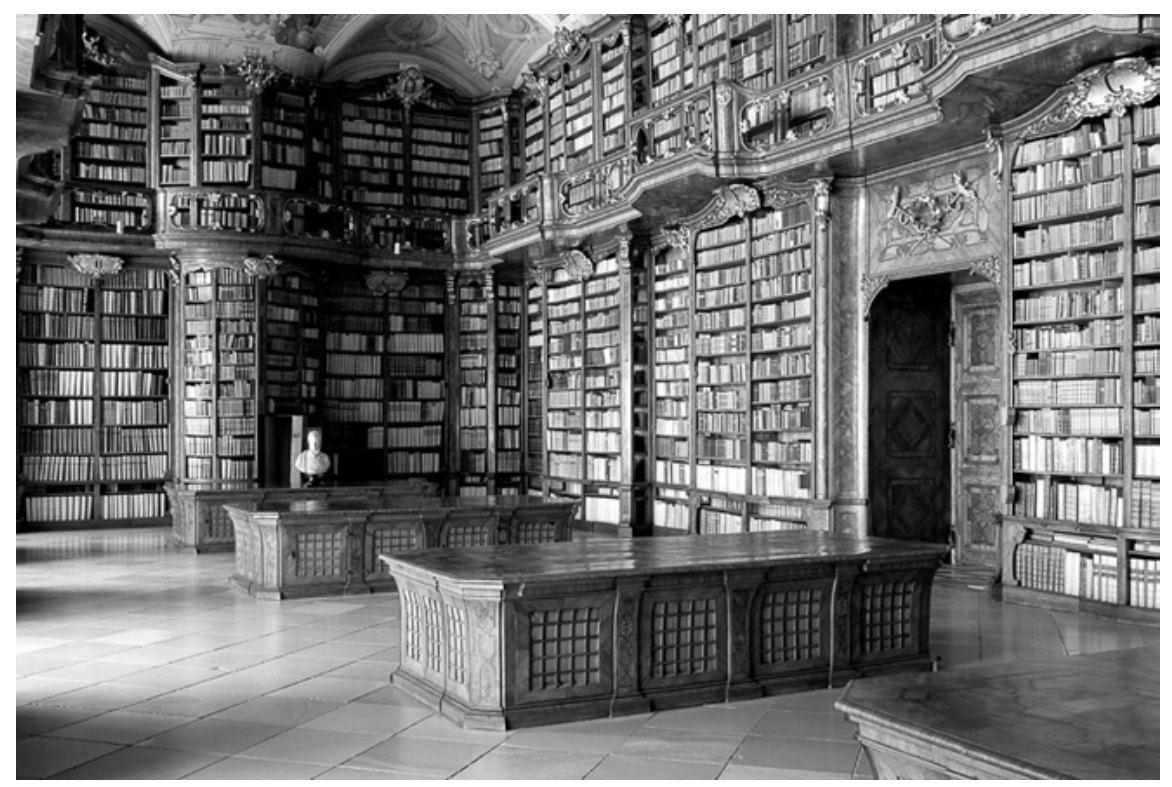

Abb. 1: Hauptsaal der Stiftsbibliothek St. Florian.

\section{Zur Charakteristik einer Stiftsbibliothek}

Klösterliche Bibliotheken und Archive sind aufgrund ihrer spezifischen historischen Entwicklung anders geprägt als entsprechende öffentliche Sammlungen. Als der St. Florianer Augustiner-Chorherr und spätere Hofarchivar Joseph Chmel 1827 von einem Reiseschriftsteller um einen Text über die Stiftsbibliothek gebeten wurde, sprach er diese Differenz an:

Ihrer Aufforderung gemäß, will ich es versuchen eine Skizze unserer Büchersammlung zu entwerfen, denn gegen den Ausdruck Bibliothek muß ich aus dem Grunde sogleich protestiren, weil man von einer Bibliothek mit Recht eine gleichmäßige Berücksichtigung aller Fächer verlangt und erwartet, was bey uns nicht der Fall ist [...]. (Chmel 1827)

Klosterbibliotheken wie St. Florian sind demnach keine Universalbibliotheken, in denen alle Fachgebiete ,gleichmäßig“ vertreten sind, und auch keine systematischen Sammlungen mit klarem Ankaufsprofil. Jahrhundertelang hätten sich die Chorherren beim Büchererwerb ausschließlich vom Aspekt der Nützlichkeit für die Seelsorge in den Stiftspfarren und für den Unterricht an der eigenen theologischen Lehranstalt leiten lassen, erläuterte Chmel weiter; diese Orientierung am Gebrauchswert könnte man immerhin als lose Sammlungsstrategie bezeichnen. 
Seit dem Ende des 18. Jahrhunderts spielten darüber hinaus die „zufälligen Liebhabereyen einzelner Stiftsmitglieder“ (Chmel 1827) eine Rolle. Gerade dieser Teil des Bücherbestands wäre aber bedeutsam und beachtenswert.

Die Stiftsbibliothek St. Florian ist in Chmels Augen ein heterogener Bestand mit Eigendynamik, der stark von Einzelpersonen geprägt ist, die bestimmte Fächer mit Liebe und Hingabe betrieben und entsprechende Spezialsammlungen hinterlassen haben.

\section{Stiftsbibliothek St. Florian}

\section{a) Geschichtlicher Überblick}

Als eigentlicher Gründer der Stiftsbibliothek gilt Propst Leopold Zehetner (16121646), der erstmals für eine gezielte Bestandsvermehrung sorgte (vgl. dazu Buchmayr 1996a). 1637 vollendete sein Bibliothekar Wolfgang Rainner den ersten Bibliothekskatalog und gab ihm den blumigen Titel Paradisus Sapientiae. Rainner teilte den Bestand in 17 Fachgebiete auf, innerhalb derer die Bücher nach Formaten geordnet waren. Ein zusätzliches Autorenregister erleichterte die Suche. Ihren größten Gönner fand die Stiftsbibliothek in Propst Johann Georg Wiesmayr (1732-1755), der den Neubau des spätbarocken Bibliothekssaales durchführte und über Agenten in vielen europäischen Städten Bücher im Gesamtwert von 11.000 Gulden erwarb. Die thematische Ordnung wurde beibehalten. Der systematische Katalog von 1746 teilte den Bestand von 15.000 Bänden in fünf Fachgebiete mit 31 Ober- und 833 Unterkapiteln auf.

Im 19. Jahrhundert bereicherten hochkarätige individuelle Sammlungen die Stiftsbibliothek, etwa jene moderner Literatur und Philosophie (Aufklärung, Romantik) von Propst Michael Ziegler oder jene der naturwissenschaftlichen Sparte durch den Chorherrn Josef Schmidberger. Hinzu kamen die Nachlässe bedeutender Historiker („St. Florianer Historikerschule“; vgl. Rehberger 1979). Deshalb sah sich Bibliothekar Albin Czerny in den 1860er-Jahren gezwungen, eine große Neuordnung vorzunehmen. Er teilte den Bestand in 15 Fachgebiete mit 36 Sparten auf und erweiterte die Bibliothek über den Hauptsaal hinaus auf sieben Nebenräume. Czerny führte den allgemeinen alphabetischen Autorenkatalog seiner Vorgänger weiter und legte zusätzlich zu jeder Sparte einen gebundenen alphabetischen Autorenkatalog an. Um Platz zu gewinnen, nahm er auch Skartierungen vor und sonderte z. B. die damals gering geschätzte barocke Periochensammlung aus.

Albin Czernys System gilt für den historischen Bestand bis 1970. Die Neuzugänge ab dem Erscheinungsjahr 1971 werden nach dem Numerus currens in 
zwei Formaten aufgestellt. Neben einem Autoren- und Schlagwortkatalog in Zettelform gibt es seit 1998 auch einen EDV-gestützten Katalog.

\section{b) Handschriften und Fragmente}

Zum Bestand der Stiftsbibliothek St. Florian gehören etwa 900 Handschriften und viele Fragmente, darunter einige für die Literaturgeschichte wichtige, die kurz erwähnt werden sollen. Das lateinische Versepos Ruodlieb aus dem 11. Jahrhundert ist nur in Fragmenten der Bayerischen Staatsbibliothek München und der Stiftsbibliothek St. Florian (Abb. 2) überliefert. Der unbekannte Autor hat erstmals im deutschsprachigen Raum eine romanartige Handlung entworfen, in deren Mittelpunkt schon das Idealbild eines christlichen Ritters stand. Das St. Florianer Fragment ist besonders wertvoll, weil es als eine unter Aufsicht des Dichters selbst angefertigte sorgfältige Reinschrift des Ruodlieb gilt (vgl. Klopsch 2010). ${ }^{1}$ Das Fragment 29 entstammt der Kaiserchronik und dürfte um 1200 geschrieben worden sein. Dem 13. Jahrhundert sind die Fragmente zum Summarium Heinrici zuzuordnen, dem umfangreichsten Glossenwerk des deutschen Mittelalters, das vom 11. bis zum 14. Jahrhundert zu den wichtigsten Wissensenzyklopädien zählte (vgl. Hildebrandt 2010). Das St. Florianer Fragment der mittelhochdeutschen Verserzählung Der arme Heinrich von Hartmann von Aue befindet sich heute in einer Berliner Bibliothek. ${ }^{2}$ Das St. Florianer Steinbuch aus dem 15. Jahrhundert und die erste deutsche Ilias-Übersetzung von Johann Baptist Rexius aus dem Jahr 1584 sind durch neuere Editionen erschlossen worden (Venosa 2001; Willing 2009). Die Edition eines Jerusalem-Reiseberichts aus dem 16. Jahrhundert ist in Vorbereitung.

Auch die Chorherren selbst haben bedeutende Handschriften hinterlassen. Altmann von St. Florian (um 1150-1221/22) gilt als erster Rechtsgelehrter Österreichs und schrieb neben einer Passio des Stiftspatrons Florian einen umfangreichen Hoheliedkommentar und zahlreiche Werke zur Kanonistik, die alle versifiziert und großteils noch nicht ediert sind (vgl. Stelzer 2010; Neschwara 1987). Einwik Weizlan von St. Florian (um 1240-1313) verfasste eine Biografie der Inklusin und Mystikerin Wilbirg, deren Beichtvater er gewesen war. Die Vita Wilbirgis ist eine der wichtigsten österreichischen Frauenbiografien des Mittelalters und eine erstrangige Quelle zur Landesgeschichte und zur Geschichte der Mystik.

1 Entdeckt wurde das Fragment im Jahr 1830 vom St. Florianer Chorherren und Historiker Jodok Stülz (vgl. Buchmayr 2014, 147-161).

2 Berlin, Staatsbibliothek, mgf 923 Nr. 7a. Der Entdecker Jodok Stülz hatte das Fragment dem Germanisten Franz Pfeiffer zur Publikation überlassen, aus dessen Nachlass es später nach Berlin gelangte. 


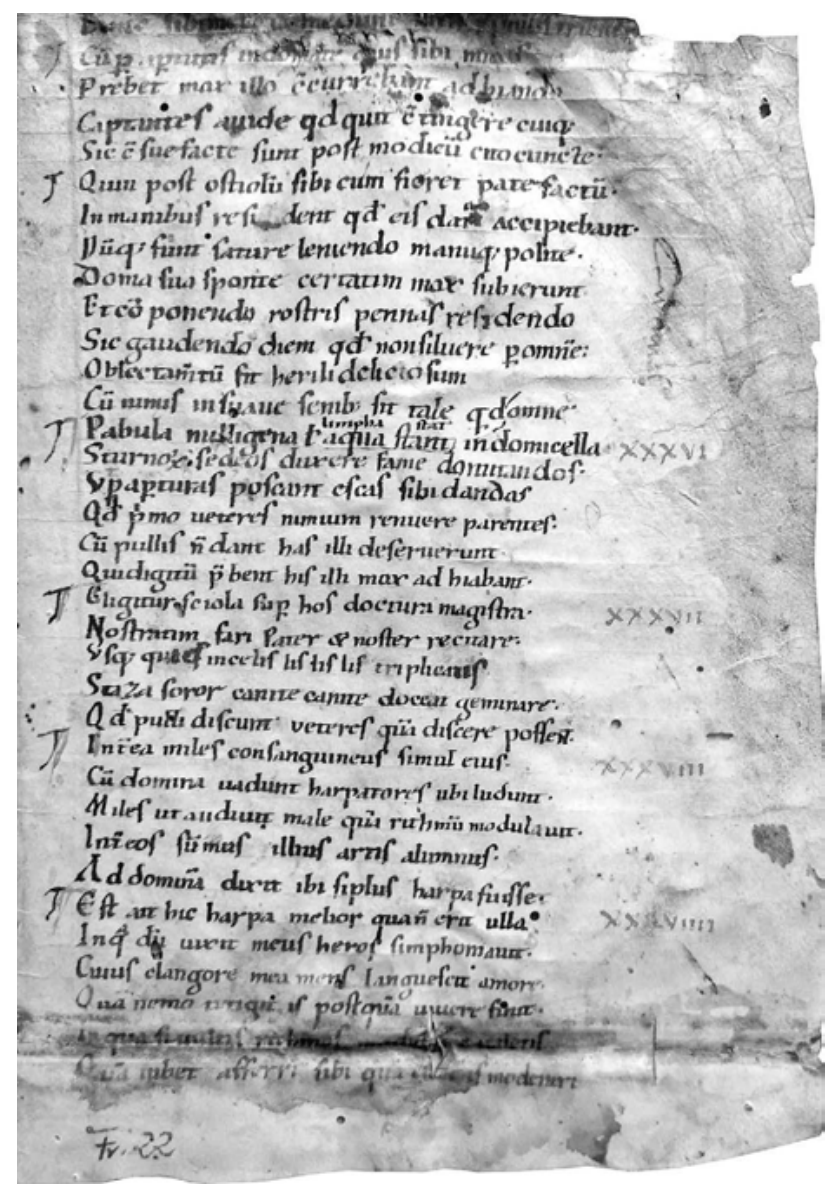

Abb. 2: Fragment des Ruodlieb aus dem Stift St. Florian (Ende 11. Jh.).

Eine zweisprachige Edition konnte auf kurz zuvor entdeckte Fragmente zurückgreifen, die Korrekturen des Autors selbst enthielten und wertvolle Rückschlüsse auf die Konzeption des Werks zuließen (vgl. Sainitzer 1999).

\section{c) Druckwerke}

Von den rund 150.000 Bänden der Stiftsbibliothek St. Florian stammen 108.000 Bände aus der Zeit vor 1900. Bei den Themengebieten dominiert nicht, wie man vermuten könnte, die Theologie allein. Theologie und Geschichte liegen mit jeweils ca. 21\% des Altbestands gleichauf, die Literatur hält bei 11\%. Zählt man allerdings die klassische Literatur der Römer und Griechen (9,5\%) mit, schließt die Literatur zur Theologie und Geschichte auf (vgl. Buchmayr 1996a). 
Die 880 Inkunabeln, die bei den Druckwerken an vorderster Stelle zu nennen sind, können über den Inkunabelzensus Österreich auf der Homepage der Österreichischen Nationalbibliothek recherchiert werden. Weniger bekannt ist ein Sonderbestand, der auf eine Liebhaberei des Stiftsbibliothekars Karl Eduard Klein (1773-1837) zurückgeht. Seine Sammlung von Frühdrucken römischer und griechischer Klassiker im Kleinformat aus exquisiten europäischen Offizinen wird in der Abteilung „Cimelien“ aufbewahrt. Später sind dieser Sparte illuminierte Handschriften, bedeutende Erstausgaben literarischer Werke, bibliophile Drucke und Werke mit kunstvollen Einbänden hinzugefügt worden.

Auch der übrige Buchbestand der Stiftsbibliothek birgt, trotz einiger Verkäufe und Verluste in den Jahren der Wirtschaftskrise zwischen den Weltkriegen und in der NS-Zeit, noch viele Kostbarkeiten. So finden sich 18 Druckwerke des großen humanistischen Schriftstellers Sebastian Brant in der Stiftsbibliothek. Bei den Exemplaren zweier seiner Drucke, den Disticha Catonis (1506) und des Facetus (1513), handelt es sich um Unikate, also um die einzigen überlieferten Exemplare dieser Ausgaben. Mit dem Jahrgang 1619 der Kölner Zeitschrift Historicae Relationes Continuatio besitzt die Stiftsbibliothek ein weiteres Unikat.

1991 fand sich bei Recherchen zum Mozart-Jubiläumsjahr eher zufällig eine Mozart-Biografie aus dem Jahr 1806, die der Forschung gänzlich unbekannt war. Im St. Florianer Katalog ist dieses anonyme Werk unter dem Titel Skizze von Mozart's Leben eingetragen gewesen und so unbemerkt geblieben (vgl. Buchmayr 1996b). ${ }^{3}$

Einzelne Druckwerke der Stiftsbibliothek sind anderen Werken beigebunden (Adligate) und deshalb gelegentlich den Bibliothekaren beim Katalogisieren entgangen. 2013 fand sich auf diese Weise die 21-seitige Perioche $\mathrm{zu}$ jenem Theaterstück, das bei der Eröffnung des Marmorsaals 1731 aufgeführt worden ist. Das Bühnenwerk lieferte anhand des historischen Perserkönigs Kyros eine Apotheose auf Kaiser Karl VI. und war genau mit dem Programm des Deckenfreskos abgestimmt (vgl. Telesko und Buchmayr 2013).

\section{d) Aktuelle Projekte}

Manche Teilsammlungen müssen lange warten, bis ihre Bedeutung erkannt und ihr Vorhandensein geschätzt wird. Ein Beispiel dafür ist die Bibliothek (samt Nachlass) des Orientalisten und Weltpriesters Hermann Stieglecker (1885-1975; Abb. 3), der als Professor an der Hauslehranstalt des Stiftes St. Florian unterrichtet hat. Aus der Kenntnis von rund 70 Sprachen vollendete er sein Lebenswerk Die

3 Bisher ist weltweit nur ein einziges weiteres Exemplar in der Wienbibliothek (Signatur A-108063) aufgetaucht. 


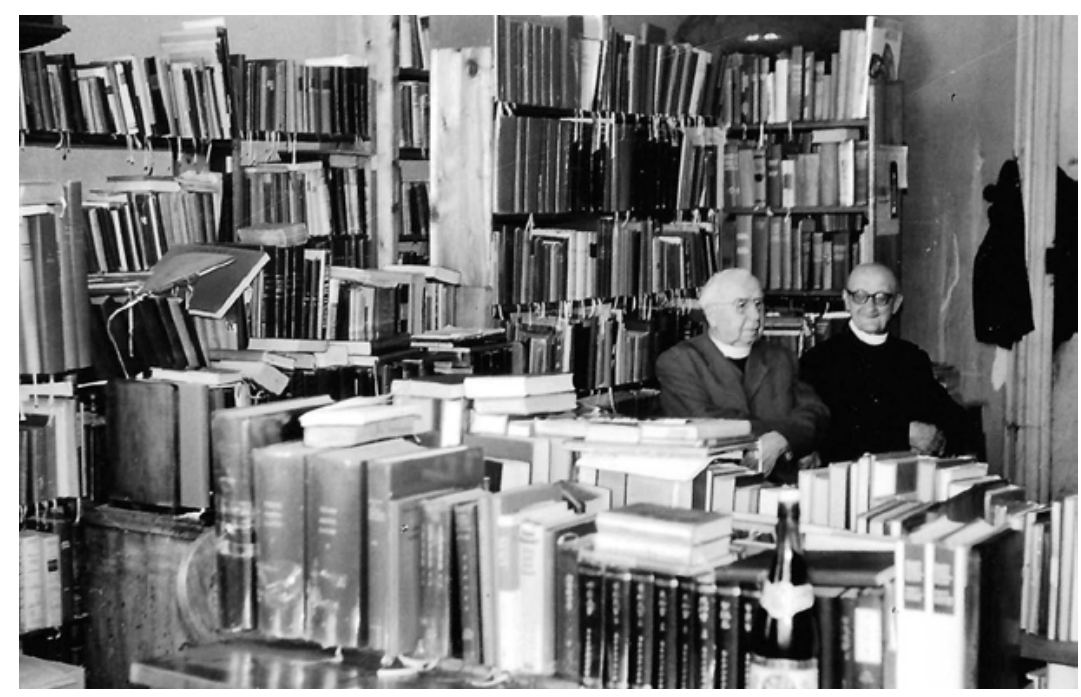

Abb. 3: Der Orientalist Hermann Stieglecker (rechts) mit Gast in seinem Wohnzimmer.

Glaubenslehren des Islam (1962), das nach seinem Tod in Vergessenheit geriet. Stieglecker ließ sich von der Intention leiten, die fremde Religion aus der Innensicht der Gläubigen darzustellen. In Zusammenarbeit mit dem Forum für Weltreligionen und dem Institut für Orientalistik der Universität Wien wird seit 2017 das geistige Erbe Stiegleckers wieder zugänglich gemacht und seine Leistung als Vorreiter des interreligiösen Dialogs gewürdigt. ${ }^{4}$ Inzwischen konnten in St. Florian zwei internationale Hermann-Stieglecker-Gedächtnistagungen abgehalten werden, deren Referate in Buchform erscheinen werden. Eine Neuauflage des genannten Hauptwerks Stiegleckers steht vor der Vollendung. Der Katalog der Bibliothek von Stiegleckers Lehrer Rudolf Geyer (1861-1929), die nach dessen Tod für die Stiftsbibliothek St. Florian erworben wurde, kann mittlerweile online abgerufen werden. ${ }^{5}$

Die Jahre 2016 und 2017 standen ganz im Zeichen der Gesamtrestaurierung des Hauptsaals der Stiftsbibliothek. Es kam zu einer sensiblen Oberflächenreinigung des Deckenfreskos von 1747, zur Erneuerung der elektrischen Leitungen und der Deckenbeleuchtung, zur Reparatur der Fenster und der Bodenfliesen und zur Restaurierung des Mobiliars und der Schnitzwerke samt ihrer Goldfassungen. In

$4 \mathrm{http} / / /$ weltreligionen.at/?/214-0-Symposien.html (25.11.2019).

5 http://www.stift-st-florian.at/stift-st-florian/stiftsbibliothek/kataloge-und-veroeffentlichungen.html (25.11.2019). Der Orientalist Cornelis van Lit hat den Katalog der 1.500 Bücher erstellt. 
den Sommermonaten 2018 und 2019 folgte die Reinigung aller Regalböden und der rund 40.000 Bücher im Hauptsaal der Stiftsbibliothek. Die dabei dokumentierten Schäden an den Büchern werden seither mithilfe von Sponsoren und Buchpaten nach Dringlichkeitsstufen restauriert. Im Zuge der Reinigungsaktion fanden sich zwei Fragmente der Riesenbibel von St. Florian (um 1140), die Ende des 16. Jahrhunderts ausgeschieden und als Bucheinbände verwertet wurden (vgl. Buchmayr und Kafka 2019).

\section{Stiftsarchiv}

Die Bedeutung des Stiftsarchivs St. Florian ist aus seinem Aufbewahrungsort abzulesen. Es war (wie die Silberkammer) von Anfang an in der Nähe der Prälatur untergebracht und wanderte bei den Umbauten, selbst beim großen Barockneubau, und den damit verbundenen Verlagerungen immer mit der Prälatur mit. Archiv und Registratur waren bis zum Ende des 18. Jahrhunderts eins und dienten rechtlich-administrativen Zwecken, also zur Dokumentation der Untertanenverhältnisse, des Besitzstands, der Schenkungen und der juristischen Privilegien. Diese Funktion des Stiftsarchivs als Herrschaftsinstrument zeigte sich auch an symbolischen Gesten. Noch im Jahr 1793 wurden dem neuen Propst bei der Amtsübergabe die Archivschlüssel und das älteste Stiftsurbar ausgehändigt. ${ }^{6}$

Im 19. Jahrhundert rückte der historische Wert der Archivalien in den Blickpunkt. Damit änderte sich auch das Ordnungsschema und die Sammlungsstrategie. Stiftsarchivar Jodok Stülz (1799-1872), einer der bedeutendsten Historiker Oberösterreichs (vgl. zuletzt Petz-Grabenbauer und Slapnicka 2010), nahm eine gründliche Skartierung vor und übergab viele Akten an die 1848 errichteten neuen Verwaltungsbehörden (Bezirksämter, Bezirksgerichte etc.). Gleichzeitig ordnete er die übrig gebliebenen Archivalien nach wissenschaftlichen Kriterien und erschloss sie durch Repertorien. Neue Sammlungen wie die Nachlässe der Chorherren kamen hinzu.

\section{a) Urkunden}

Das Stiftsarchiv St. Florian besitzt rund 2.400 Urkunden auf Pergament, die chronologisch geordnet sind. Die Reihe beginnt mit der ältesten Originalurkunde des Landes Oberösterreich aus dem Jahr 1002. Alle Urkunden bis zum Jahr 1600

6 Stiftsarchiv St. Florian, Stiftsakten, 1793 September 10./11. 
konnten in Zusammenarbeit mit dem Verein Icarus 2006 digitalisiert werden und sind online über die Plattform Monasterium einsehbar (www.monasterium.net).

\section{b) Stiftsakten (allgemeine Akten)}

Dieser heterogene Bestand, zu dem $u$. a. auch Papierurkunden gehören, ist nach dem Vorbild der Urkunden ebenfalls chronologisch geordnet und umfasst 154 Kartonschuber bis zum Jahr 1900. Der bereits erwähnte Stiftsarchivar Jodok Stülz hat ein dreibändiges Register zu den Urkunden und Stiftsakten (Chronologisches Register aller im Stiftsarchive zu St. Florian vorhandenen Urkunden und Schriften) erstellt, das von Stiftsarchivar Karl Rehberger (1934-2018) auf Computer übertragen und ergänzt wurde. Es finden sich bedeutende Schriftstücke darunter, etwa eine frühe Chronik der Stadt Linz aus dem Jahr 1626 (vgl. Haider 1968).

Mit der Jahrhundertwende 1900 erfolgte ein Einschnitt und die chronologische Ordnung von Stülz wurde aufgegeben. Seither werden die Akten entweder nach dem Provenienzprinzip eingestellt, d. h. nach den Stiftsbetrieben, aus denen sie stammen, oder nach Themen und Sachgruppen wie „Anton Bruckner“, „Kunst“, „Restaurierung“, „Orgel“ usw.

\section{c) Handschriften (archivalische)}

Das Stiftsarchiv verwahrt rund 370 gebundene archivalische Handschriften, die zum Großteil von Stiftsarchivar Jodok Stülz verzeichnet und durch ein Register erschlossen worden sind (Verzeichniß der im Stiftsarchive zu St. Florian vorhandenen gebundenen Schriften und Acten). Dazu kommen noch etwa 145 gebundene archivalische Handschriften aus den Stiftspfarren, in der Hauptsache Zehentund Dienstbücher sowie Urbare, die aus besitzrechtlichen Überlegungen angelegt worden sind. Alle Handschriften sind in Zusammenarbeit mit dem Oberösterreichischen Landesarchiv 2004 mikroverfilmt worden. Die 90 Filmrollen mit den ca. 50.000 Aufnahmen können auch im Landesarchiv eingesehen werden.

Unter den Handschriften ragt das älteste Kopialbuch Österreichs von 1276/1360 (Hs. 101 b) hervor, das einzigartige kolorierte Porträts der Aussteller der Urkunden enthält. Bemerkenswert ist auch die Kirchweihchronik (Hs. 101 a), die im Zusammenhang mit der Weihe der gotischen Stiftskirche im Jahr 1291 vom späteren Propst Einwik Weizlan (1295-1313) angelegt wurde. Das älteste Stiftsurbar stammt aus dem Jahr 1378 (Hs. 107 a).

Über Nachlässe sind auch externe Handschriften in das Stiftsarchiv gekommen, so etwa Linzer Stadtordnungen des 16. bis 18. Jahrhunderts (Hs. 62) aus dem Besitz des Linzer Advokaten Johann Karl Seyringer. Für die Hausgeschichte von Bedeutung sind die mehr als 100, großteils eigenhändigen Handschriften des geschichtsbewussten Propstes Johann Georg Wiesmayr (1732-1755). Einige von 
ihnen hat Stiftsarchivar Karl Rehberger durch Faksimileausgaben (Series Praelatorum, Hs. 79 a, und Topographia Florianensis, Hs. 78) oder durch sorgfältige Editionen (Advocatus fidelis, Hs. 95, und Flebile pro memoria, Hs. 71) im Linzer Wagner-Verlag zugänglich gemacht.

\section{d) Akten der Stiftspfarren}

Die sehr heterogenen Akten und Schriftstücke zu den 33 Stiftspfarren („Pfarrschachteln“) sind in etwa 300 Kartonschachteln abgelegt und geben Einblick in deren religiöses und wirtschaftliches Leben. Hinzu kommen ca. 295 Kartonschuber mit Kirchen(bau)rechnungen, die ebenfalls nach Stiftspfarren geordnet sind. In nächster Zukunft ist aufgrund der kirchlichen Entwicklung mit der Übernahme ganzer Stiftspfarrarchive zu rechnen.

\section{e) Rechnungs- und Finanzwesen}

Die allgemeinen Baurechnungen sind in 65 Schubern abgelegt und umfassen den Zeitraum von 1677 bis 1835. Der barocke Neubau des Stiftes ist darin sehr umfassend dokumentiert. Kammereirechnungen sind aus den Jahren 1625 bis 1799 erhalten geblieben (214 Bände). Bedeutend für die Baugeschichte wie für die Ausstattungsgeschichte sind die diversen Rechnungsbücher, in denen die Ankäufe von Büchern und Kunstgegenständen von Linzer Märkten (und anderen Städten) dokumentiert sind. Die Marktrechnungen umfassen 18 Schuber zu den Jahren 1652 bis 1784, die Kastenrechnungen 19 Schuber (1669 bis 1869).

\section{f) Wirtschaftsakten aus den Stiftsbetrieben}

Diese Akten wurden jeweils aus der Registratur bzw. aus der Zentralverwaltung übernommen. Es handelt sich um rund 1.000 Rechnungsbücher der Stiftsverwaltung vom 17. bis ins 20. Jahrhundert. In der Hauptsache sind es Rentamtsbücher, also jährliche Journale der Wirtschaftsdirektion, und Rechnungsbücher der einzelnen Stiftsbetriebe (Küche, Brauerei, Kelleramt usw.). Oft sind darin wertvolle Hinweise verborgen. So ließen sich anhand von Gästelisten bei den Akten der Stiftsküche die Aufenthalte des Komponisten Anton Bruckner im Stift St. Florian genau dokumentieren (vgl. Buchmayr 2011-2014). Ein Besuch Michael Haydns im Jahr 1773 ist nur über einen Honorarvermerk im Ausgabenbuch des damaligen Propstes nachweisbar (vgl. Buchmayr 2006). Dieser Einzelband erinnert gleichzeitig an die vielen nicht erhaltenen Rechnungsbücher aus der Prälatur, die nicht im Nachhinein aussortiert, sondern von Vornherein nie an das Stiftsarchiv übergeben worden sein dürften. 


\section{g) Nachlässe}

Jahrhundertelang wurden nach dem Tod von Ordensmitgliedern keine persönlichen Schriftstücke oder Dokumente archiviert. Ab dem 17. Jahrhundert legte man beim Tod von Pröpsten die vorgeschriebenen Gesamtinventare über das Stiftsvermögen an. Erst im frühen 19. Jahrhundert setzte sich das Bewusstsein durch, dass die private Korrespondenz und die schriftlichen Aufzeichnungen von wissenschaftlich oder literarisch tätigen Mitbrüdern von Bedeutung und erhaltenswert sind. Die systematische Errichtung von Nachlässen begann im Stift St. Florian mit zwei angesehenen Gelehrten unter den Chorherren, dem Theologen Franz Josef Freindaller (1753-1825) und dem Historiker Franz Kurz (1771-1843).

Eine Anregung des Österreichischen Staatsarchivs zur Nachlasserschließung führte in St. Florian 2013 zu einer entsprechenden Initiative. Innerhalb von zwei Jahren konnten die elf wichtigsten Nachlässe des 19. Jahrhunderts katalogisiert und in säurefreie Umschläge und Schachteln (104 Stück) umgelagert werden. Dabei waren u. a. 8.500 Briefe nach Absendern zu ordnen und einzeln zu katalogisieren.

Die Nachlässe der Chorherren enthalten neben eigenen Werken Briefe von berühmten Persönlichkeiten aus Wissenschaft, Politik, Kunst und Literatur, darunter regionale Autorinnen wie Caroline Pichler, Enrica von Handel-Mazzetti und Hedda Wagner, aber auch von internationalen Größen wie Joseph von Hormayr, Joseph von Hammer-Purgstall, August Heinrich Hoffmann von Fallersleben und Theodor Mommsen. Im Nachlass von Ernst Marinelli (1824-1887) fand sich unerwartet ein unbekannter Brief Adalbert Stifters, der 2005 in einer Sonderausstellung zu Stifters 200. Todestag präsentiert wurde (vgl. Buchmayr 2007). Zwei unbekannte Originalbriefe Anton Bruckners kamen im Nachlass Gottfried Schneidergrubers (1879-1941) zum Vorschein (vgl. Buchmayr 2013).

$\mathrm{Zu}$ den originellsten Teilsammlungen innerhalb der Nachlässe zählt das 1890 begonnene Reisejournal in Form von Ansichtskarten des Chorherrn Christoph von Chiusole (1875-1943), das 61 Bände mit 20.000 Ansichtskarten umfasst und chronologisch geordnet ist. ${ }^{7}$

\section{h) Publikationen}

Aus der Vielzahl von Veröffentlichungen zum Stiftsarchiv sind zwei größer angelegte Werke hervorzuheben. In den Jahren 1963, 1978 und 1979 wurden von Stu-

7 Die 16.700 Ansichtskarten mit Oberösterreich-Motiven aus der topografischen Sammlung sind 2008 digitalisiert worden und können online betrachtet werden: http://www.doris.at/themen/ geschichte/hist_ansichten.aspx (25.11.2019). 
denten und Absolventen des Instituts für österreichische Geschichtsforschung in Wien die wichtigsten Bestände des Stiftsarchivs St. Florian auf Bezüge zur Stadtgeschichte von Linz hin durchgesehen und in Regestenform beschrieben. Gegenstand der Forschung waren die Urkunden, die Stiftsakten (bis zum Jahr 1849), die wichtigsten Archivhandschriften, die Akten zu den Stiftspfarren Ebelsberg, Ansfelden und Pulgarn sowie die Marktrechnungen. Die Linzer Regesten (vgl. Fleck et al. 1982) aus dem Stiftsarchiv St. Florian umfassen vier Bände.

Auf der Basis langjähriger eigener Aufzeichnungen hat der schon erwähnte Stiftsarchivar Karl Rehberger eine umfangreiche Bibliografie zum Stift St. Florian erstellt, das Publikationen bis zum Jahr 2015 berücksichtigt (vgl. Rehberger, Chr. und J. Wunscheim 2006, 2011 und 2016).

\section{Bibliothek und Archiv im Selbstverständnis der Chorherren}

Über den Stellenwert, den Bibliothek und Archiv im heutigen Selbstverständnis der Augustiner-Chorherren des Stiftes St. Florian einnehmen, gibt ihr aktuelles Leitbild Auskunft. Dort heißt es im Kapitel „Der Ort unseres Lebens und Wirkens“: „Das Stift bietet Möglichkeit für die Auseinandersetzung mit gesellschaftlich aktuellen Themen, die Bibliothek stellt einen Anreiz dar für wissenschaftliches Arbeiten, besonders in den Bereichen Theologie und Geschichte.“ Dieser Grundsatz wird an späterer Stelle noch konkretisiert: „Die Pflege von Wissenschaft, Kunst und Kultur verstehen wir als einen Teil unseres Gesamtverständnisses von Seelsorge. ${ }^{\text {8 }}$

Stiftsarchiv und Stiftsbibliothek dienen somit, wie die übrigen Sammlungsbereiche, als Bewahrer des kulturellen Erbes der Vergangenheit und als Plattform für geistige Auseinandersetzungen in der Gegenwart. Dass es sich dabei um keine bloßen Lippenbekenntnisse handelt, zeigen die permanenten Restaurierungsprojekte ebenso wie die vermehrten Aktivitäten im Ausstellungs-, Kunstvermittlungs- und Führungsbereich, aber noch viel mehr Forschungsprojekte wie jenes zum Orientalisten Hermann Stieglecker, das vom Stift St. Florian personell und finanziell mitgetragen wird.

8 http://www.stift-st-florian.at/die-chorherren/die-st-florianer-chorherren/leitbild.html (25.11.2019). 


\section{Literaturverzeichnis}

Buchmayr, Friedrich. „St. Florian. Bibliothek des Augustiner-Chorherrenstiftes“. Handbuch der historischen Buchbestände in Österreich. Hg. Österreichische Nationalbibliothek. Bd. 3. Bearb. v. Wilma Buchinger und Konstanze Mittendorfer. Hildesheim, Zürich, New York: Olms-Weidmann, 1996a. 267-272.

Buchmayr, Friedrich. „Vom Bürgerschreck zum ,rechtschaffensten Mann““. Eine unbekannte Mozart-Biographie aus dem Jahr 1806. Mozart-Jahrbuch (1996b): 73-84.

Buchmayr, Friedrich. „Michael Haydn und das Stift St. Florian“. Oberösterreichische Heimatblätter 60 (2006): 14-21.

Buchmayr, Friedrich. „Die Pilgerreise nach Jerusalem. Adalbert Stifters Beziehungen zum Stift St. Florian“. Stifter und die Stifterforschung im 21. Jahrhundert. Hg. Alfred Doppler, Johannes John, Johann Lachinger und Hartmut Laufhütte. Tübingen: Niemeyer, 2007. 97-116.

Buchmayr, Friedrich, Karl Rehberger und Friedrich Simader. Die Riesenbibel von St. Florian. Graz: Akad. Dr.- und Verl.-Anst., 2008.

Buchmayr, Friedrich. „Prälatengang Nr. 5. Anton Bruckner als Gast im Stift St. Florian“. Bruckner-Jahrbuch (2011-2014): 7-44.

Buchmayr, Friedrich. „Zwei unbekannte Briefe Anton Bruckners“. Streifzüge. Beiträge zur oberösterreichischen Musikgeschichte. Hg. Klaus Petermayr. Bd. 3. Linz: Oberösterreichisches Volksliedwerk, 2013. 177-185.

Buchmayr, Friedrich. Ein Ort von Welt. 13 europäische Reisende erleben das Stift St. Florian. Regensburg: Schnell und Steiner, 2014.

Buchmayr, Friedrich, und Clemens Kafka. „Zwei neue Fragmente der Riesenbibel von St. Florian“. Codices manuscripti \& impressi 114/115 (2019): 1-8.

Chmel, Joseph. Joseph Chmel, Skizze der Bibliothek zu St. Florian [1827]. Stiftsbibliothek St. Florian. Handschrift XI 541 A.

Fleck, Christine et al. (Bearb.). Linzer Regesten. B X 1-4: Stiftsarchiv St. Florian. Linz: Archiv der Stadt Linz, 1982 (Typoskript).

Haider, Siegfried. „Eine alte Chronik der Stadt Linz“. Historisches Jahrbuch der Stadt Linz (1968): 321-335.

Hildebrandt, Reiner. „Summarium Heinrici“. Die deutsche Literatur des Mittelalters. Verfasserlexikon. Hg. Kurt Ruh, Gundolf Keil, Werner Schröder, Burghart Wachinger und Franz Josef Worstbrock. Bd. 9. Unver. Neuausg. der 2. Aufl. Berlin, New York: de Gruyter, 2010. 510-519.

Holter, Kurt. „Bibliothek und Archiv: Handschriften und Inkunabeln“. Die Kunstsammlungen des Augustiner-Chorherrenstiftes St. Florian. Hg. Veronika Birke und Rotraud Bauer. Wien: Schroll, 1988. 29-92.

Holzinger, Johann, und Friedrich Buchmayr (Hg.). Augustiner-Chorherrenstift St. Florian. Regensburg: Schnell und Steiner, 2009.

Klopsch, Paul. „Ruodlieb“. Die deutsche Literatur des Mittelalters. Verfasserlexikon. Hg. Kurt Ruh, Gundolf Keil, Werner Schröder, Burghart Wachinger und Franz Josef Worstbrock. Bd. 8. Unver. Neuausg. der 2. Aufl. Berlin, New York: de Gruyter, 2010. 395-400.

Korth, Thomas. Stift St. Florian. Die Entstehungsgeschichte der barocken Klosteranlage. Nürnberg: Verlag Hans Carl, 1975. 
Korth, Thomas. „Baugeschichte“. Augustiner-Chorherrenstift St. Florian. Hg. Johann Holzinger und Friedrich Buchmayr. Regensburg: Schnell und Steiner, 2009a. 8-13.

Korth, Thomas. „Die Prunkräume und das Treppenhaus“. Augustiner-Chorherrenstift St. Florian. Hg. Johann Holzinger und Friedrich Buchmayr. Regensburg: Schnell und Steiner, 2009b. 43-52.

Petz-Grabenbauer, Maria, und Harry Slapnicka. „Jodok Stülz“. Österreichisches Biographisches Lexikon. Hg. Österreichischen Akademie der Wissenschaften unter der Leitung von Leo Santifaller. Bd. 13. Graz: Böhlau, 2010. 443.

Neschwara, Christian. „Altmann von St. Florian“. Juristen in Österreich. 1200-1980. Hg. Wilhelm Brauneder. Wien: Orac, 1987. 18-19.

Reisinger, Ferdinand, und Katharina Brandes. „St. Florian“. Die bestehenden Stifte der Augustiner-Chorherren in Österreich, Südtirol und Polen. Hg. Floridus Röhrig. Klosterneuburg: M \& C, 1997. 337-384.

Rehberger, Karl. „Die St. Florianer Historikerschule. 100 Jahre Geschichtsschreibung“. Ostbairische Grenzmarken 21 (1979): 144-154.

Rehberger, Karl, Augustinus Franz Kropfreiter und Helmut Kögler. Große Orgel in der Stiftskirche St. Florian. Ried im Innkreis: Kunstverlag Hofstetter, 1998.

Rehberger, Karl, Christiane Wunschheim und Johannes Wunschheim. Bibliographie zur Geschichte des Stiftes Sankt Florian. Linz: Oberösterreichisches Landesarchiv, 2006 [mit Ergänzungsbänden 2000-2010 (Linz, 2011) und 2011-2015 (Linz, 2016)].

Rehberger Karl. „Der hl. Florian und die Anfänge des Stiftes St. Florian“. Augustiner-Chorherrenstift St. Florian. Hg. Johann Holzinger und Friedrich Buchmayr. Regensburg: Schnell und Steiner, 2009. 6-8.

Sainitzer, Lukas. Die Vita Wilbirgis des Einwik Weizlan. Kritische Edition und Übersetzung von Lukas Sainitzer. Linz: Oberösterreichisches Landesarchiv, 1999.

Schmidt, Gerhard. Die Malerschule von St. Florian. Beiträge zur süddeutschen Malerei zu Ende des 13. und im 14. Jahrhundert. Graz: Böhlau, 1962.

Stelzer, Winfried. „Altmann von St. Florian“. Die deutsche Literatur des Mittelalters. Verfasserlexikon. Hg. Kurt Ruh, Gundolf Keil, Werner Schröder, Burghart Wachinger und Franz Josef Worstbrock. Bd. 1. Unver. Neuausg. der 2. Aufl. Berlin, New York: de Gruyter, 2010. 308-310.

Telesko, Werner, und Friedrich Buchmayr. „Der ,Marmorsaal' im Augustiner-Chorherrenstift St. Florian. Die Verherrlichung des Türkensiegers Kaiser Karl VI. im Lichte schriftlicher und bildlicher Quellen“. Jahrbuch des Oberösterreichischen Musealvereines - Gesellschaft für Landeskunde 158 (2013): 211-258.

Venosa, Elena Di. Il Lapidario di Sankt Florian. Edizione sinottica dei codici Sankt Florian XI 37 e Berlino Germ. Fol. 944. Milano: CUEM, 2001.

Willing, Antje (Hg.). Johannes Baptista Rexius. Ilias Homeri teutsch. Mit synoptischem Abdruck der „Ilias“-Übertragung Lorenzo Vallas. Berlin: Schmidt, 2009. 